06

\title{
Идентификация свойств полимерных композитных материалов в пространстве их ротационно-вязкостных характеристик
}

\author{
(C) А.Г. Нагиев, ${ }^{1}$ В.В. Садыхов, ${ }^{2}$ У.М. Гашимова ${ }^{1}$ \\ ${ }^{1}$ Сумгаитский государственный университет, \\ AZ 5008 Сумгаит, Азербайджан \\ ${ }^{2}$ Институт математики и механики НАН Азербайджана, \\ AZ1141 Баку, Азербайджан \\ e-mail: alinagiev@gmail.com
}

Поступило в Редакцию 26 февраля 2018 г.

В окончательной редакции 5 июня 2019 г.

Принято к публикации 28 августа 2019 г.

Найден эффект сильной корреляции между кривыми течения жидко-пластичных или разбавленных полимерных композиций и качеством готового материала. Показано, что глубина корреляции и информативность существенно зависят от конструкции турбины, создающей ротацию для вискозиметрического исследования композита. Предложен метод анализа результатов экспериментов, который основан на вычислении начальных моментов до третьего порядка от полученных кривых течения, эффективный с точки зрения прогноза достижимости заданных качественных показателей.

Ключевые слова: ротационно вязкостная характеристика полимеров, прогноз качества композитных материалов, параметризация кривых течения неньютоновских жидкостей, отображения в пространстве моментов.

DOI: $10.21883 / J T F .2020 .02 .48817 .85-18$

\section{Введение}

Технология производства композитных материалов, как известно, включает процессы смешения в растворе, в расплаве, а также адиабатическую экструзию пластичной массы с неньютоновским характером течения. Сложные реологические свойства неньютоновских сред представляют важную область исследований в физическом материаловедении. Проявления аномальной вязкости в ряде жидко-пластичных составов, обнаружение степенных законов изменения тангенциальных напряжений от скорости сдвига при знакопеременном параметре степени и многие особенности, наблюдающиеся в кривых течения, стимулируют исследования, направленные на решение проблемы заранее заданных качеств полимерно-композитных материалов (ПКМ) - важной области экспериментального материаловедения [1-3]. Перечень параметров качества производимой продукции, подлежащих прогнозу, довольно обширен. Существование корреляций между качеством производимой продукции и параметрами вискозиметрии, эволюционирующими в динамике формирования композита, представляет важный вопрос для изучения. Возможность экспресс-анализа молекулярной массы полимеров по их характеристической вязкости - давно известный факт (уравнение Марка-Куна-Хаувинка [4]), который, хотя и упрощенно, но, по сути, отражает наличие указанной корреляции. В то же время имеющийся к настоящему времени огромный экспериментальный материал [5-7] убеждает в более значительной информативности ротационных вискозиметрических характеристик в задачах косвенного оценивания, прогноза и управления качеством производимой продукции.
В ряде работ, например в [8], приведена кривая текучести, которая, выражая специфические свойства исследуемого авторами работы материала, в то же время обладает характерными чертами, наблюдаемыми во множестве других экспериментальных данных ротационной вискозиметрии. Характерная перевернутая $S$-образная кривая, связывающая сдвиговую скорость с силами сопротивления деформации, имеет в середине выраженный пологий участок. К объяснению этой особенности, встречающейся в широком наборе исследуемых материалов, разными исследователями привлечены процессы взаимодействия как молекулярного уровня [3], так и уровня образующихся глобул, конгломератов в микро-, мезо- и макромасштабах [7]. Однако наряду с существованием качественно общих характеристик значительно более высока роль специфических сторон физических явлений, которые отражаются на характеристиках течения [1,9]. Именно глубокая специфичность характера кривых течения исследуемых композитов дает веские аргументы в пользу возможности создания прогнозирующей системы ротационного анализа как альтернативе сенсорного контроля готовой продукции.

Методы вискозиметрического анализа (BА) в большинстве случаев могут способствовать адекватной оценке тех или иных показателей качества приготавливающихся композитных материалов. Поиск новых технологий в развитии ВА целесообразно вести в двух направлениях: в области конструкционного оформления элементов, задающих условия ротации, и в направлении переработки экспериментальной реологической информации. 


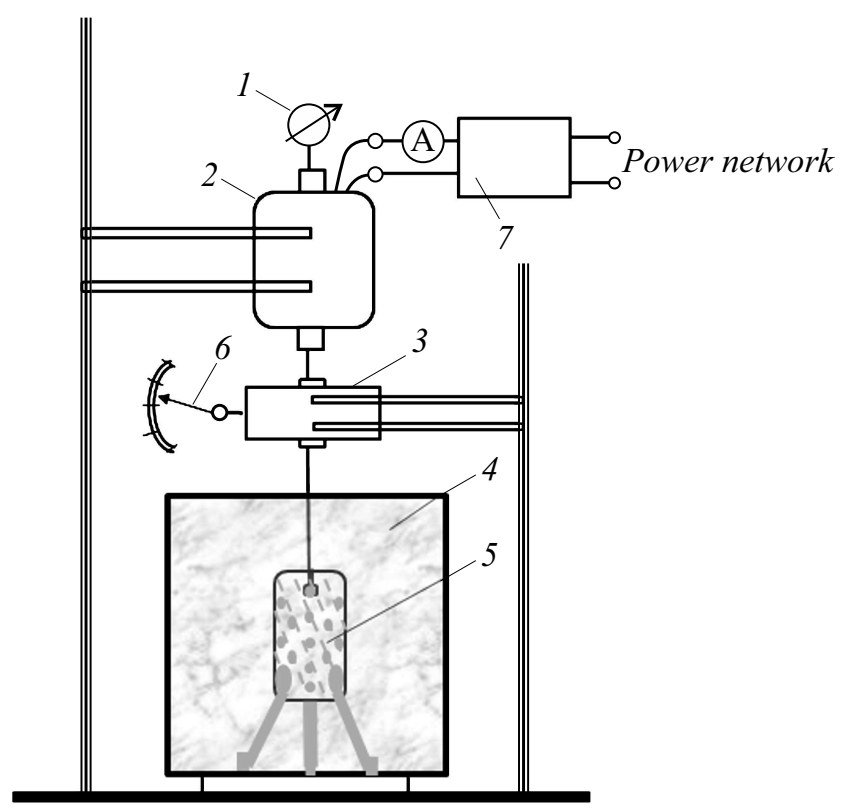

Рис. 1. Лабораторная установка для получения ротационной электромеханической характеристики привода, работающего на вязкую нагрузку.

\section{Вискозиметрическая установка с рельефными поверхностями статора и ротора с варьируемой скоростью ротации}

Принципиальная схема предлагаемого варианта лабораторной установки ротационного исследования вязкой среды приведена на рис. 1. Основное ее отличие от стандартных схем создания вискозиметров заключается в специальной конструкции цилиндра и ротора, создающих изменения скорости сдвига в определенном диапазоне. Принципом фиксирования касательного напряжения является расчетное значение вращательного момента двигателя постоянного тока, приравненное к моменту вязкого сопротивления среды в стационарных условиях.

Принцип измерения аналогичен стандартным вискозиметрам и заключается в следующем. В бак, наполненный испытуемым образцом материала, помещена турбина, движимая электроприводом постоянного тока 2. Якорная цепь двигателя питается от источника стабилизированного напряжения 7. Для измерения скорости вращения ротора использован тахометр 1. Для варьирования скорости вращения предусмотрено устройство бесступенчатой передачи 3 (вариатор) с рычагом управления 6 . Амперметр, включенный в силовую цепь якоря, служит для определения текущей (мгновенной) механической мощности, рассеиваемой в вязкой среде в результате трения.

Механическая мощность, затрачивающаяся на преодоление сил вязкого сопротивления, вычисляется по следующим соотношениям [10]:

$$
P_{2}=M \omega ; \quad P_{2}=P_{1} \eta=I U \eta,
$$

где $M$ - вращательный момент (в стационарном режиме приравненный моменту сопротивления нагрузки), $\omega$ - угловая скорость, $P_{1}-$ подведенная мощность; $P_{2}$ - полезная мощность; $\eta-$ коэффициент полезного действия, вычисляемый с учетом КПД как двигателя, так и суммарных потерь вариатора и других движущихся механических частей. Учитывая соотношения (1), можно записать

$$
M=\frac{I U}{\omega} \eta .
$$

На основании этого соотношения построение электромеханической характеристики привода с нагрузкой вязкого сопротивления сводится к варьированию коэффициента передачи вариатора и фиксированию силы тока в якорной цепи двигателя. Посредством проведения последовательных экспериментов с варьированием фиксируется величина момента сопротивления $M$. Далее строится зависимость между скоростью вращения и механическим моментом.

В отличие от традиционной конструкции ротационных вискозиметров разработанная конструкция имеет цилиндр и ротор с рельефными поверхностями (рис. 2). Вращаясь внутри наполненного герметичного бака, ротор создает усилие, выталкивающее жидкость внутри полости цилиндра, в результате которого через отверстия с низа цилиндра имеет место некоторый (незначительный) поток. Сложная гидродинамическая обстановка, создающаяся приводом такой конструкции, формирует функцию момента сопротивления, определенным образом зависящую от скорости вращения ротора. Это сопротивление характеризуется тем, что оно является следствием

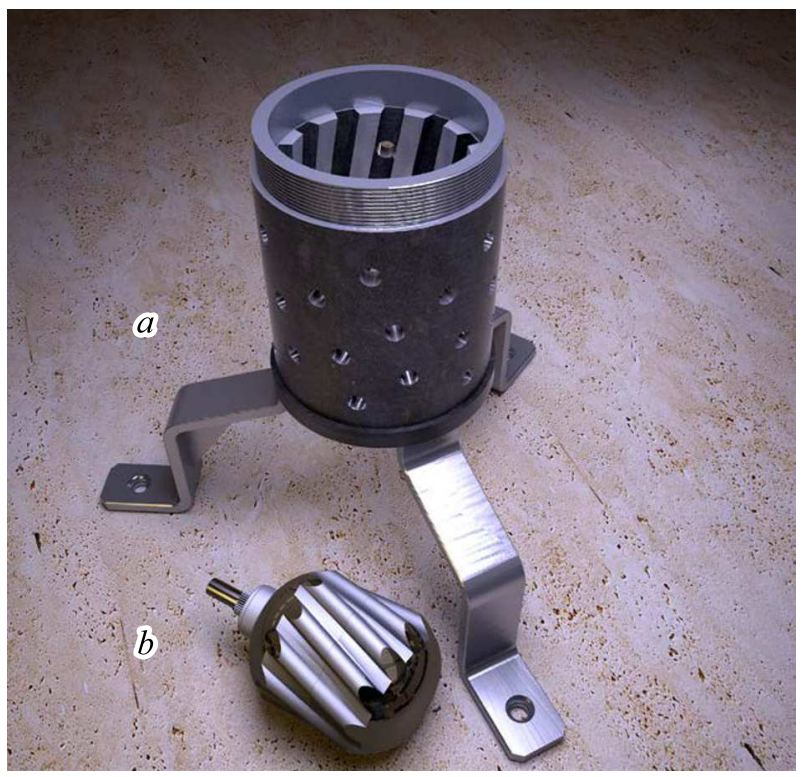

Рис. 2. Ротор $(a)$ и статор $(b)$ лабораторной установки ротационного исследования вязкой среды. 

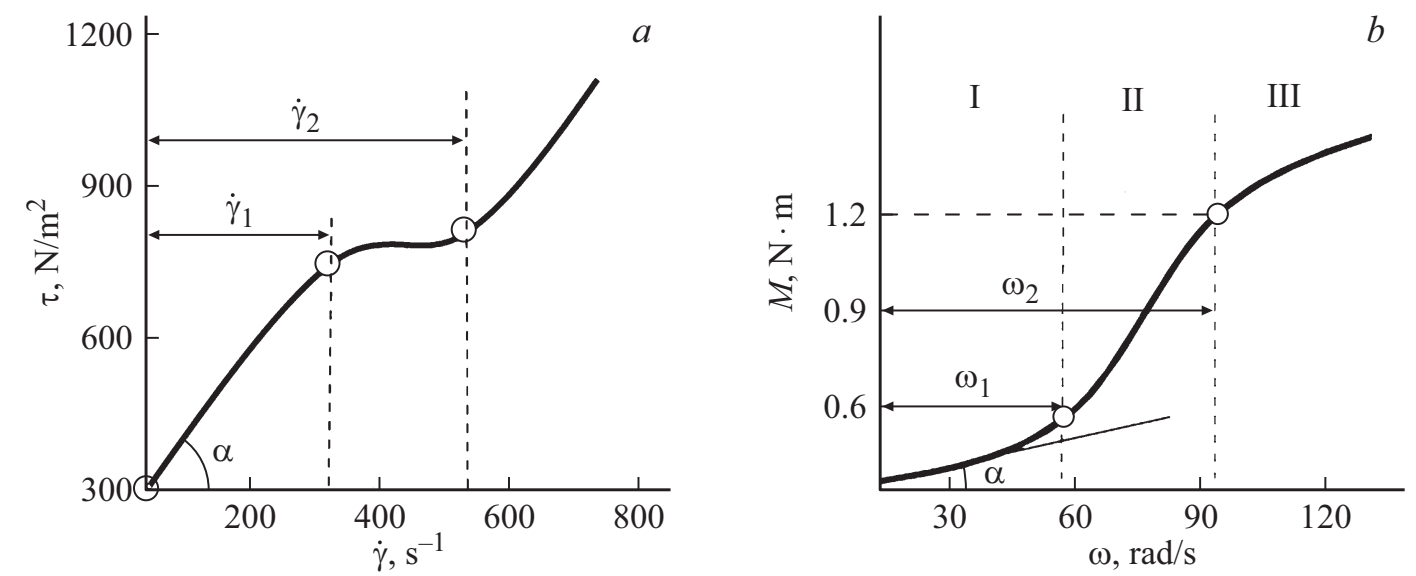

Рис. 3. Разные виды и характеристические параметры ротационно-вискозиметрической кривой для вязкой среды: $a-$ экспериментальная зависимость касательных напряжений от скорости сдвига [9]; $b$ - экспериментальная кривая изменения момента вязкого сопротивления от скорости ротации в среде эпоксидной смолы.

не только молекулярных сил взаимодействия, но и сил реакции частиц, агрегированных в конгломераты разных масштабных уровнях. Соотношение этих сил является функцией скорости ротации.

На рис. 2 показана сложная геометрия статора и ротора привода. Измерение должно проводиться в стационарных условиях с выдержкой времени, требующегося для завершения переходных процессов. Поэтому в целях снижения момента инерции ротор изготавливается в виде пустотелой детали. В зависимости от испытуемого материала диаметры отверстий и другие геометрические размеры могут изменяться.

\section{Вискозиметрическое исследование и анализ кривых течения}

На рис. 2, а показан использованный в экспериментах статор с внутренним диаметром 8.7 и $5.6 \mathrm{~mm}$. Диаметр ротора составляет $7.6 \mathrm{~mm}$, высота $4.2 \mathrm{~mm}$. Именно такая конструкция и размеры привода оказались более информативными при снятии характеристики электромеханической системы при нагрузке вязкого сопротивления материалов с вязкостью $12-25 \mathrm{~Pa} \cdot \mathrm{s}$, в частности, исследовались эпоксидные смолы марок ЭД-20 и ЭД-16 без введения каких-либо наполнителей. Температуру среды поддерживали $25^{\circ} \mathrm{C}$. В ряде экспериментов ее меняли в интервале $(25-65)^{\circ} \mathrm{C}$. Объем бака, наполненного эпоксидной смолой, составлял 121.

На рис. 3, $а$ представлена экспериментальная кривая, которая получена авторами работы [8] при измерении реологии мазей на стандартном ротационном вискозиметре „Реотест-2“, основанном на создании условий вязкого трения в среде между двумя коаксиальными, равномерно вращающимися цилиндрами. Рис. $3, b$ представляет результат проведенного нами эксперимента. Данный эксперимент описан в координатах: „угловая частота вращения ротора - момент вязкого сопро-

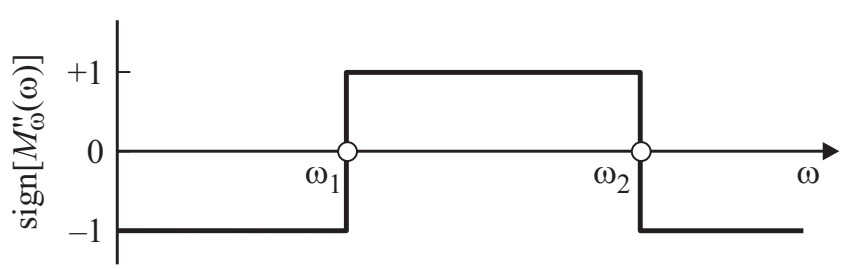

Рис. 4. Функция знака $z=\operatorname{sign}\left[M_{\omega}^{\prime \prime}(\omega)\right]$, фиксирующая координаты точек перегиба в кривой течения. Экспериментальная кривая изменения момента вязкого сопротивления от скорости ротации в среде эпоксидной смолы.

тивления“, тогда как кривая рис. 3, a построена как зависимость тангенциального напряжения от скорости сдвига, которая является общепринятой в реологических исследованиях вязких сред. На графике отмечены три участка с соответствующими тремя граничащими точками. Расположения этих точек на кривой определяют соответственно три величины, использующиеся для параметризации исследуемой функции: $\alpha-$ угол наклона (начальная крутизна) ньютоновского участка кривой; $\omega_{1}, \omega_{2}-$ абсциссы точек перегиба в характеристике. Так, дважды дифференцируя данные функции, в частности кривую на рис. $1, a$, получим соответствующую функцию знака $z=\operatorname{sign}\left[M_{\omega}^{\prime \prime}(\omega)\right]$, вид которой представлен на рис. 4.

Полученная функция может быть эффективной при параметризации кривых течения, характеризующихся наличием двух точек перегиба. В ряде опытнолабораторных исследований, проводимых для изучения свойств конкретных композитных материалов на основе эпоксидных смол, использование координат этих точек $\omega_{1}, \omega_{2}$ и начальной крутизны характеристики $\alpha$ оказалось достаточно эффективным для ряда задач идентификации.

При проведении данного экспериментального анализа наибольший интерес представляет оценка глубины 

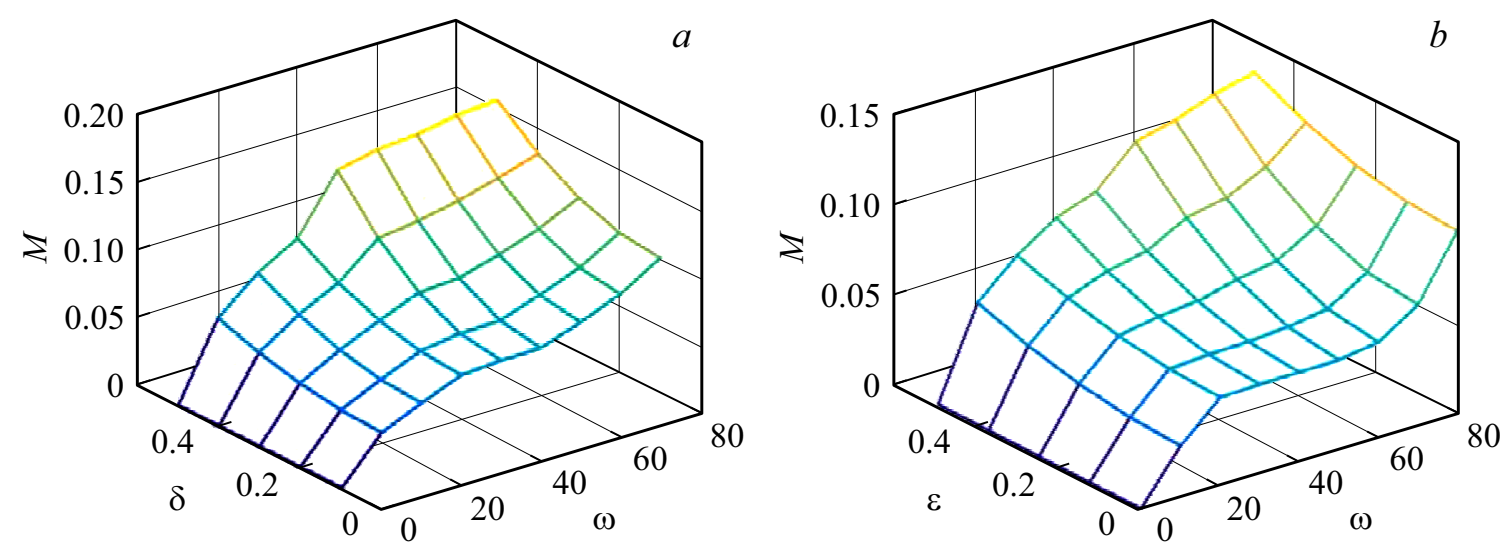

Рис. 5. Зависимости момента сопротивления от скорости вращения перемешивающего устройства композиции, состоящей из порошка древесины (дуб, зерна размером $<200 \mu$ ) и эпоксидной смолы, разбавленной на ацетоне: $a-$ при $\delta=\{0.1 ; 0.2 ; 0.3 ; 0.4\}$, $\varepsilon=0.1 ; b-$ при $\varepsilon=\{0.1 ; 0.2 ; 0.3 ; 0.4\}, T=25^{\circ} \mathrm{C}$.

корреляции между вектором $\boldsymbol{\xi}=\left\{\alpha, \omega_{1}, \omega_{2}\right\}$ и тем или иным показателем качества продукции $R$, относительно которого ставится задача прогнозирования. С этой точки зрения целесообразно использование статистической оценки результатов серии экспериментов в виде следующей формулы, так называемой выборочной чувствительности $R$ к вариации вектора $\xi$ :

$$
\begin{aligned}
k^{2}= & \frac{1}{N} \sum_{i=1}^{N}\left[\left(\frac{\bar{r}-R_{i}}{\bar{\alpha}-\alpha_{i}}\right)^{2}+\left(\frac{\bar{r}-R_{i}}{\bar{\omega}_{1}-\omega_{1 i}}\right)^{2}\right. \\
& \left.+\left(\frac{\bar{r}-R_{i}}{\bar{\omega}_{2}-\omega_{2 i}}\right)^{2}\right],
\end{aligned}
$$

где $\bar{r}, \bar{\alpha}, \bar{\omega}_{1}, \bar{\omega}_{2}-$ выборочные средние значения во множестве параметров, $N$ - число экспериментов.

Проведенные эксперименты с использованием эпоксидных смол без каких-либо наполнителей позволили вывести ряд важных заключений. Прежде всего, установлено, что на характер электромеханической кривой сильно влияет на конструкцию турбины. Очевидно, что, по сути, получаемые характеристики представляли собой описания экспериментов на установке с турбиной предлагающейся конструкции. При этом не наблюдалось влияние объема бака, наполненного исследуемым вязким материалом, если он не меньше величины 3-41. Результатами экспериментов с эпоксидными смолами без каких-либо наполнителей подтверждено, что в большинстве экспериментов функция $z=\operatorname{sign}\left[\tau_{\bar{\gamma}}^{\prime \prime \prime}(\bar{\gamma})\right]$ имеет две точки переключения знака с разными расстояниями в расположении абсцисс. Данный факт оказался полезным аргументом приемлемости параметризации кривых течения в пространстве признаков $\xi=\left\{\alpha, \omega_{1}, \omega_{2}\right\}$.

Однако в ряде результатов испытаний материалов был получен иной характер кривой течения, не допускающий идентификацию в указанном пространстве векторов. В экспериментальном исследовании композиции, состоящей из эпоксидной смолы с древесномучными наполнителями в разных концентрациях ацето- на, получены кривые, не содержащие характерные точки перегиба. В связи с этим более эффективным оказался предлагающийся нами второй вариант параметризации кривых течения, основанный на использовании начальных моментов, вычисленных для них.

Пусть для функции $M=f(\omega) ; \omega \in[0, \Omega]$ (экспериментальной кривой течения) определены начальные моменты $n$-го порядка в следующем виде:

$$
\mu^{(i)}=\frac{1}{\Omega^{i}} \int_{0}^{\Omega} \omega^{i} f(\omega) d \omega ; \quad i=\overline{0, n},
$$

где $i$ - порядок момента, $\Omega$ - концевая точка интервала варьирования угловой скорости. Точность (качество) параметризации кривых течения, очевидно, определена числом вводимых (наибольшим порядком) моментов $n$.

На рис. 5 показаны результаты двух серий опытов по изучению влияния температуры и степени разбавленности исследуемого материала (композита с древесномучным наполнителем) растворителем, в качестве которого использовался нами ацетон. Объемное соотношение „смола:порошок“ взято 1:2. На оси ординат отмечены моменты вязкого сопротивления. На одной из абсцисс отмечены угловые скорости вращения $\omega$, на другой - температура и объемное соотношение веществ $\varepsilon$ (разбавитель/смола). На рис. $5, b$ на координатной оси $\delta$ отмечены дискретно задаваемые температуры:

$$
\delta(\lambda)=T_{0}+\frac{\left(T_{\max }-T_{0}\right)}{T_{\max }} \frac{\lambda}{L} ; \quad \lambda=\overline{1, L},
$$

где $L$ - число шагов дискретизации температуры, $T_{\min }, T_{\max }$ - крайние точки температурного диапазона эксперимента.

Целью эксперимента являлась проверка методики получения оценок чувствительности исследуемых характеристик к изменениям состава и физических свойств вязких сред (полимерных композитных материалов), а также прогностической способности этих характеристик 
(качества продукции). Поставлен вопрос экспериментального изучения свойств вязкой массы: эпоксидная смола марки ЭД-20+дубовая мука с размером частиц $<200 \mu+$ растворитель (ацетон) в разных концентрациях.

Для оценки чувствительности, информативности, а также прогнозирующей способности показанных на графиках экспериментальных зависимостей осуществлена их аппроксимация полиномами:

$$
M=\varphi_{k}\left(\omega, \varepsilon_{k}\right), M=\varphi_{\lambda}\left(\omega, \delta_{\lambda}\right), k=\overline{1, K} ; \lambda=\overline{1, L},
$$

где $\varepsilon_{k}, \delta_{\lambda}$ - параметры, $K$ - число опытов с варьированием концентрации разбавителя.

Использованы начальные моменты нулевого $\mu^{(0)}$, первого $\mu^{(1)}$ и второго $\mu^{(2)}$ порядков, вычисляемые по формуле (2). По четырем экспериментальным данным вычислены чувствительности функций (3) по параметрам $\varepsilon$ и $\delta$ :

$$
\begin{aligned}
k_{\varepsilon}^{2}= & \frac{1}{K} \sum_{k=1}^{K}\left[\left(\frac{\bar{\mu}^{(0)}-\mu_{k}^{(0)}}{\bar{\varepsilon}-\varepsilon_{k}}\right)^{2}+\left(\frac{\bar{\mu}^{(1)}-\mu_{k}^{(1)}}{\bar{\varepsilon}-\varepsilon_{k}}\right)^{2}\right. \\
& \left.+\left(\frac{\bar{\mu}^{(2)}-\mu_{k}^{(2)}}{\bar{\varepsilon}-\varepsilon_{k}}\right)^{2}\right], \\
k_{\varepsilon}^{2}= & \frac{1}{L} \sum_{\lambda=1}^{K}\left[\left(\frac{\bar{\mu}^{(0)}-\mu_{\lambda}^{(0)}}{\bar{\delta}-\delta_{\lambda}}\right)^{2}+\left(\frac{\bar{\mu}^{(1)}-\mu_{\lambda}^{(1)}}{\bar{\delta}-\delta_{\lambda}}\right)^{2}\right. \\
& \left.+\left(\frac{\bar{\mu}^{(2)}-\mu_{\lambda}^{(2)}}{\bar{\delta}-\delta_{\lambda}}\right)^{2}\right] .
\end{aligned}
$$

Анализ полученных результатов показал высокую чувствительность параметров идентификации $\xi_{\mu}=\left\{\mu^{(0)}, \mu^{(1)}, \mu^{(2)}\right\}$ к изменениям $\varepsilon$ и $\delta$ относительно испытуемой эпоксидной смолы.

\section{Оценки идентифицирующей способности

ротационно-вискозиметрических
характеристик

Согласно данной методике, вопрос идентификации (прогноза) качества непосредственно связывался с исследованиями разделимости экспериментальных данных в пространстве параметров ротационных характеристик. В частности, использовалась параметризация кривых на основе начальных моментов по формуле (2). Планируя две серии опытов с композитом (смола ЭД20 + дубовая мука) с разбавлением исследующийся массы ацетоном и проводя эксперименты при разных температурах, мы исходили из того, что каждое множество условий эксперимента, обладая интервальной близостью по отношению к вязкости среды, в то же время представляло результат влияния различных физических эффектов. В частности, для формирования

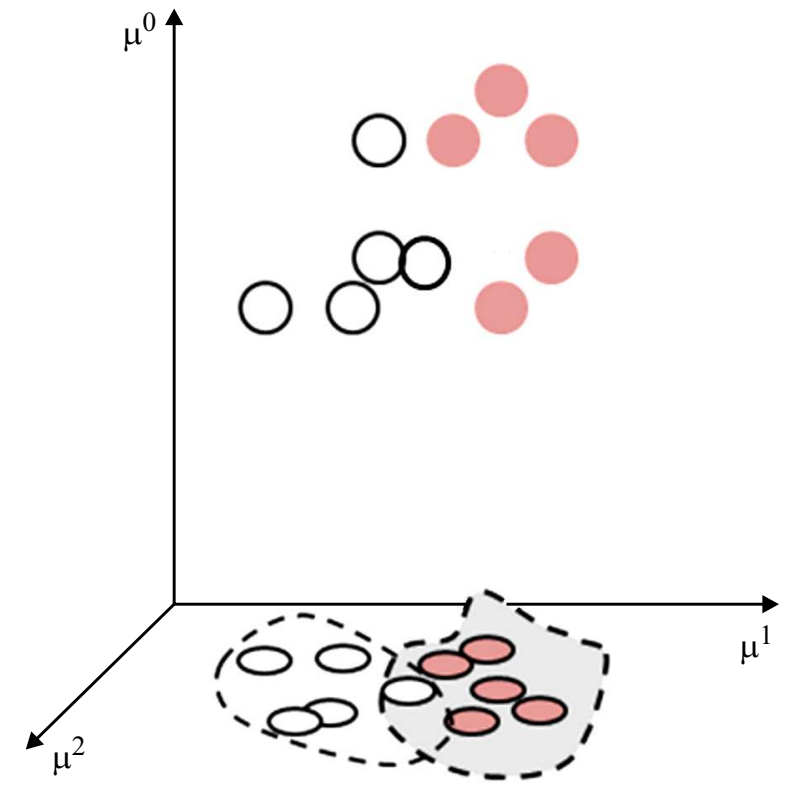

Рис. 6. Изображение разделимости кривых течения в пространстве моментов.

опытных образцов смесей с вязкостными параметрами, покрывающими заданный интервал, менялась температура или смесь разбавлялась растворителем. В результате, получив почти одинаковый диапазон средней вязкости для каждой серии опытов с вариациями температуры $\delta$ или доли растворителя $\varepsilon$, мы смогли поставить вопрос о разделимости опытных данных в пространстве параметров $\xi_{\mu}=\left\{\mu^{(0)}, \mu^{(1)}, \mu^{(2)}\right\}$ согласно их происхождениям. Важно то, что положительный ответ на этот вопрос может характеризовать именно высокую идентифицирующую способность изучаемой характеристики. Степень разделимости оценивалась по суммарному евклидовому расстоянию между точками каждой группы эксперимента - группы с меняющейся температурой и группы с различными концентрациями растворителя.

В таблице приведены координаты точек, которые отображают два множества кривых ротационного исследования эпоксидных смол с пятью разными степенями разбавления ацетоном и тем же количеством кривых, отличающихся различием температурных условий эксперимента.

На рис. 6 показаны точки в пространстве начальных моментов нулевого, первого и второго порядков, параметризующие экспериментальные кривые течения.

Факт высокой разделимости экспериментальных данных в пространстве параметров $\mu^{(i)} ; i=\overline{0,2}$, существование корреляции между качественными параметрами и изучаемыми реологическими характеристиками, представляют веские аргументы целесообразности постановок широкого спектра задач управления заданным качеством ПКМ. Стоит отметить, что современный уровень метрологической оснащенности исследо- 
Экспериментальные кривые течения, представленные в трехмерном пространстве моментов, полученные при разных температурах и степенях разбавленности эпоксидной смолы ацетоном

\begin{tabular}{|c|c|c|c|c|c|}
\hline \multicolumn{5}{|c|}{ Температура $T=25^{\circ} \mathrm{C}$} & \multirow{3}{*}{$\begin{array}{l}\text { Суммарное расстояние } \\
\text { между центрами групп }\end{array}$} \\
\hline \multirow{2}{*}{ Отношение $\varepsilon=V_{s} / V_{r}$} & \multicolumn{3}{|c|}{ Значения моментов } & \multirow{2}{*}{$\begin{array}{c}\text { Суммарное расстояние между } \\
\text { точками одной группы }\end{array}$} & \\
\hline & $\mu^{(0)}$ & $\mu^{(1)}$ & $\mu^{(2)}$ & & \\
\hline 0.0 & 0.0615 & 0.0431 & 0.0322 & \multirow{5}{*}{0.102} & \multirow{12}{*}{0.230} \\
\hline 0.04 & 0.0518 & 0.0351 & 0.0227 & & \\
\hline 0.08 & 0.0478 & 0.0332 & 0.0225 & & \\
\hline 0.12 & 0.0461 & 0.0330 & 0.0216 & & \\
\hline 0.16 & 0.0442 & 0.0316 & 0.0145 & & \\
\hline \multicolumn{5}{|c|}{ Степень разбавленности смолы $\varepsilon=0.08$} & \\
\hline Температура $\delta,{ }^{\circ} \mathrm{C}$ & $\mu^{(0)}$ & $\mu^{(1)}$ & $\mu^{(2)}$ & & \\
\hline 35 & 0.0517 & 0.0360 & 0.0239 & \multirow{5}{*}{0.097} & \\
\hline 45 & 0.0467 & 0.0327 & 0.0268 & & \\
\hline 55 & 0.0447 & 0.0324 & 0.0188 & & \\
\hline 65 & 0.0440 & 0.0265 & 0.0108 & & \\
\hline 70 & 0.0416 & 0.0243 & 0.0103 & & \\
\hline
\end{tabular}

вательских лабораторий позволяет поставить задачу экспериментального изучения зависимости механических параметров вязкоупругих свойств ПКМ от характеристик течения. Например, методами динамического механического анализа по методике трехточечного изгиба [5] могут быть измерены три параметра для прочности ПКМ: модуль упругости $\left(E^{\prime}\right)$, потери при изгибе $\left(E^{\prime \prime}\right)$, а также тангенс угла механических потерь $(\operatorname{tg} \delta)$. В такой постановке вопроса относительно прогнозирующей модели качества ПКМ целесообразным оказалось бы введение выражения, связывающего названные показатели качества с вектором моментов $\xi_{\mu}$ от кривых течения при неизменных условиях всех прочих факторов:

$$
\begin{aligned}
& E^{\prime}=a_{10} \mu^{(0)}+a_{11} \mu^{(1)}+a_{12} \mu^{(2)}, \\
& E^{\prime \prime}=a_{20} \mu^{(0)}+a_{21} \mu^{(1)}+a_{22} \mu^{(2)}, \\
& \operatorname{tg} \delta=a_{30} \mu^{(0)}+a_{31} \mu^{(1)}+a_{32} \mu^{(2)} .
\end{aligned}
$$

где $a_{i j} ; \quad i=\overline{1,3} ; \quad j=\overline{0,2}$ коэффициенты, аппроксимирующие совокупность экспериментальных данных.

\section{Заключение}

Экспериментальные исследования вязких сред связующих матриц композитных материалов с наполнителем и без него на базе проектированной нами установки ротационной вискозиметрии позволили прийти к следующим заключениям:
1. Экспериментально установлено наличие тесной взаимосвязи между параметрами вискозиметрии и параметрами исследуемого качества при заданной конструкции ротационной установки. Данный факт служит веской аргументацией того, что поиск оптимального диапазона варьирования скорости ротации должен быть связан c задачей обеспечения максимальной информативности опытных данных. Проведение оптимизации в этом направлении должно составлять неотъемлемую часть задачи раннего мониторинга качества выпускающейся продукции.

2. Для ряда вязких сред характерны перевернутые $S$ образные реограммы с наличием двух точек перегиба. Подобная качественная особенность кривых течения оказывается благоприятной в решении задач идентификации и контроля. Однако более универсальным и достаточно точным оказывается параметризация реограмм, основанная на вычислении начальных моментов третьего порядка. Оказалось, что именно этот способ представления реограмм в трехмерном пространстве их параметров обеспечивает наибольшую степень разделимости исследуемых образцов композитных материалов по отношению к условиям проведения эксперимента.

3. Идентификация качества композитных материалов, направленная на разработку рецептуры композитов с заранее заданным качеством и на решение проблемы предварительного мониторинга качества, может быть успешно решена методами обработки данных активного эксперимента. 


\section{Финансирование работы}

Работа выполнена при поддержке Фонда развития науки при Президенте Азербайджанской Республики (проект EİF/MQM/Elm-Təhsil-1-2016-1(26)).

\section{Конфликт интересов}

Авторы заявляют, что у них нет конфликта интересов.

\section{Список литературы}

[1] Boi S., Mazzino A., Pralits J.O. // Phys. Rev. E. 2013. Vol. 88. N 3. P. 033007. doi/10.1103/PhysRevE.88.033007

[2] Fall A., Huang N., Bertrand F., Ovarlez G., Bonn D. // Phys. Rev. Lett. 2008. Vol. 100. N 3. P. 018301. doi/10.1103/PhysRevLett.100.018301

[3] Xiangwu Zhang, Yi Pan, Jianfeng Cheng, Xiaosu Yi. // J. Mater. Sci. 2000. Vol. 35. N 18. P. 4573-4581. doi.org/10.1023/A:1004845426786

[4] Mark H. Der Feste Korper, Leipzig, 1938 / P.F. Onyon, „Viscometry“, in P.W. Allen, Techniques of Polymer Characterization, Butterworths, London, 1959.

[5] Москалюк О.А., Самсонов А.М., Семенова И.В., Смирнова В.Е., Юдин В.Е. // ЖТФ. 2017. Т. 87. Вып. 2. С. 266-270.

[6] Редников С.Н., Султанов И.Н. // Вестник ЮУрГУ. Серия: Машиностроение, 2011. № 11. С. 38-42.

[7] Jang D.S., Zhang W.L., Choi H.J. // J. Mater. Sci. 2014. Vol. 49. N 20. P. 7309-7316. doi.org/10.1007/s10853-0148443-5

[8] Камаева С.С., Поцелуева Л.А., Сафиуллин Р.С., Бейг С.М., Лебедева С.М. // Фундаментальные исследования. 2005. № 7. C. 89-92.

[9] Hickey C.M.D., Bickerton S. // J. Mater. Sci. 2013. Vol. 48. N 2. P. $690-701$.

[10] Вольдек А.И., Попов В.В. Электрические машины. СПб.: Питер, 2008. 320 c. 